\title{
Variabilidad genética poblacional de la trucha Yaqui (Oncorhynchus sp.) en la región de Mesa Tres Ríos, Sonora, México
}

\author{
Population genetic variability of the Yaqui trout (Oncorhynchus sp.) in the region of \\ Mesa Tres Rios, Sonora, Mexico
}

\begin{abstract}
Carlos A. Ballesteros-Córdova', Alejandro Varela-Romero²*, José M. Grijalva-Chon², Reyna A. Castillo-Gámez², Faustino Camarena-Rosales ${ }^{3}$ y Gorgonio Ruiz-Campos ${ }^{3}$

Licenciatura en Biología, Universidad de Sonora, Blvd. Luis Donaldo Colosio s/n, Col. Centro, Hermosillo, Sonora, 83000, México.

2 Departamento de Investigaciones Científicas y Tecnológicas de la Universidad de Sonora, Blvd. Luis Donaldo Colosio s/n, Col. Centro, Hermosillo, Sonora, 83000, México.

3 Facultad de Ciencias Universidad Autónoma de Baja California, Carr. Tijuana-Ensenada Km 103, Ensenada, Baja California, 22870, México.
\end{abstract}

\section{RESUMEN}

La trucha Yaqui pertenece a un grupo de truchas nativas no descritas del género Oncorhynchus del Noroeste de México. Estudios morfológicos y moleculares postulan a la trucha Yaqui como una nueva especie exclusiva para las cuencas de los ríos Yaqui, Mayo y Casas Grandes y, evolutivamente muy cercana a la trucha arcoíris, Oncorhynchus mykiss. El conocimiento de su historia natural es escaso. Se estudió la variabilidad genética poblacional y su estatus de conservación en su distribución en Sonora en la región de Mesa Tres Ríos. Se muestrearon seis arroyos de aguas frías (localidades) en la región de Mesa Tres Ríos en dos vertientes geográficas en la búsqueda de estructura genética poblacional. Se analizaron 61 secuencias completas de la región control del ADN mitocondrial. Se obtuvieron nueve haplotipos, tres de ellos se encontraron en ambas vertientes de las localidades del área estudiada. Los resultados mostraron una baja diferenciación genética poblacional (Fst $=0.08035$ ) y un moderado número de migrantes por generación (Nem = 5.72), que resulta en una falta de estructura poblacional y sustenta la hipótesis de una sola población de trucha Yaqui en las vertientes muestreadas de la región de Mesa Tres Ríos, Sonora.

Palabras clave: Región control, Estructura genética, trucha Yaqui, Noroeste de México

\section{ABSTRACT}

Yaqui trout belongs to a group of undescribed native trouts of the Oncorhynchus genus in the Northwest Mexico. Morphological and molecular studies postulate the Yaqui trout as a new species exclusively inhabiting the Yaqui, Mayo and Casas Grandes rivers basins, and with a very close evolutionary relationship to the rainbow trout, Oncorhynchus mykiss. Knowledge of its natural history is scarce. We studied the population genetic variability and its conservation status in its distribution in Sonora in the Mesa Tres Ríos region. Six cold water streams (localities) were sampled in the Mesa Tres Ríos region in two geographic slopes in the search for population genetic structure. We analyzed 61 complete sequences of the mitochondrial DNA control region. Nine haplotypes were obtained, three of them found in both slopes of the localities in the studied area. Results showed a low population genetic differentiation ( $F s t=0.08035)$, and a moderate number of migrants per generation (Nem $=5.72)$, which results in a lack of population structure, and supports the hypothesis of a single population of Yaqui trout in the sampled watersheds from the Mesa Tres Ríos region, Sonora.

Keywords: Control region, Genetic structure, Yaqui trout, Northwest Mexico

\section{INTRODUCCIÓN}

Las truchas son peces de la familia Salmonidae que se encuentran representadas por 70 especies repartidas en 10 géneros (Hendrickson et al., 2002; Mayden et al., 2010). Habitan en zonas de aguas frías en regiones montañosas, originalmente en el hemisferio Norte y actualmente en casi todo el mundo debido a su uso en acuacultura por su alto valor comercial (FAO, 2005). En México, el género Oncorhynchus tiene amplia distribución y representa a la totalidad de las truchas nativas de México. Ocupan hábitats acuáticos naturales de zonas serranas, donde la trucha arcoíris, Oncorhynchus mykiss ha sido introducida para su cultivo en ambientes naturales (Hendrickson et al., 2002; Espinosa-Pérez et al., 2007; Varela-Romero y Hendrickson, 2009; Mayden et al., 2010).

Sólo dos especies de truchas nativas se han descrito actualmente para México, la trucha dorada Mexicana, Oncorhynchus chrysogaster (Needham y Gard, 1964), endémica a las cuencas de los Ríos Fuerte, Sinaloa y Culiacán en la Sierra Madre Occidental (SMO) (Miller, 1950; Needham y Gard, 1964; Behnke, 1991; 2002; Hendrickson et al., 2003; Ruiz-Campos et al., 2003; Miller et al., 2005) y la trucha de Nelson, Oncorhynchus mykiss nelsoni Evermann, 1908, una subespecie endémica a la vertiente oeste de la Sierra San Pedro Mártir en Baja California (Evermann, 1908; Nelson, 1921; Snyder, 1926; Smith, 1991; Ruiz-Campos y Pister, 1995; Miller et al., 2005). 
El resto de la biodiversidad de truchas nativas para México está compuesta por un complejo que incluye un número indeterminado de nuevas especies de diferentes linajes que habitan de norte a sur en la SMO (Behnke, 2002; Hendrickson et al., 2002, 2006; Mayden, 2004; Camarena-Rosales et al., 2008; Varela-Romero y Hendrickson, 2009; Mayden et al., 2010; Escalante et al., 2014; Abadía-Cardoso et al., 2015, 2016). Este complejo de especies no descritas representa nuevos genomas nativos para la ciencia, que la mayoría aún se conservan debido a la inaccesibilidad de las localidades donde habitan y a la carencia de descripciones formales para su protección legal (Hendrickson et al., 2002; Ruiz-Campos et al., 2003; Mayden, 2004; Mayden et al., 2010).

Entre ellas, la trucha Yaqui pertenece a un linaje norteño de este complejo de especies que de acuerdo con varios autores habita las cuencas de los Ríos Yaqui y Mayo en Sonora y, Casas Grandes en Chihuahua (Mayden et al., 2010; Escalante-Sánchez et al., 2014; Abadía-Cardoso et al., 2015, 2016). Los impactos más drásticos sobre las poblaciones de truchas nativas en estas cuencas hidrológicas son la introducción de peces exóticos, la construcción de aserraderos y caminos (Hendrickson et al., 2003; Varela-Romero y Hendrickson, 2009). Muchos arroyos tributarios donde habitan las truchas nativas muestran efectos de erosión, sedimentación, contaminación y abatimiento de los flujos de agua ocasionada por el desarrollo de actividades extractivas (Miller et al., 2005; Minckley y Marsh, 2009). El mal manejo de la trucha arcoíris exótica en forma de criaderos en el área de distribución de las truchas nativas, amenaza la integridad genética de la trucha Yaqui y del resto de su complejo de especies en la SMO, principalmente por introgresión genética, competencia e incluso depredación (Ruiz-Campos et al., 2003; Varela-Romero y Hendrickson, 2009; Escalante et al., 2014).

Los estudios moleculares iniciales del complejo de especies de truchas de la SMO mostraron que la trucha Yaqui estaba muy relacionada con la trucha arcoíris exótica, O. mykiss (Loudenslager et al., 1986; Nielsen, 1996; 1997; Nielsen et al., 1997; 1998). Sin embargo, los estudios morfológicos y moleculares posteriores (Ruiz-Campos et al., 2003; Camarena-Rosales et al., 2008) reconocieron la identidad específica de la trucha Yaqui con muestras de las cuencas de los ríos Yaqui y Mayo y, sugieren que esta especie es nativa en su distribución en la SMO.

Posteriormente, estudios moleculares de la trucha Yaqui y este complejo de especies donde se analizan secuencias de fragmentos de la región de control, polimorfismos de longitud de fragmentos de restricción (RFLP por sus siglas en inglés), microsatélites y polimorfismos nucleotídicos simples (SNPs por sus siglas en inglés), muestran una gran diversidad genética para este complejo de truchas de la SMO y reconocen cuatro linajes genéticos, que podrían considerarse a nivel de especie para la trucha dorada mexicana (O. chrysogaster), las truchas de los ríos Yaqui, Casas Grandes y Mayo, las truchas de los ríos Piaxtla y San Lorenzo y las truchas de los ríos Presidio y Baluarte (Nielsen y Sage, 2001; Camarena-Rosales et al., 2008; De Los Santos-Camarillo, 2008; Mayden et al.,
2010; Abadía-Cardoso et al., 2015). Recientemente, García-De León et al. (en preparación) por medio de microsatélites identifica ocho Unidades Evolutivamente Significativas (ESU por sus siglas en inglés) en la SMO, una para las truchas norteñas de las cuencas de los ríos Yaqui, Mayo y Casas Grandes; una en el norte del Río Conchos; otra para O. chrysogaster; dos para las truchas de los ríos San Lorenzo y Piaxtla, dos entre los ríos Presidio, Baluarte y Acaponeta y una en la Península de Baja California al interior de este complejo, las propone como un instrumento indispensable para iniciar prácticas de conservación, reconoce la carencia de nombres científicos y las amenazas de extinción para este complejo de truchas nativas mexicanas.

Sin embargo, los estudios sobre distribución, el estado de su conservación genética y la estructura genética poblacional de la trucha Yaqui en Sonora son inexistentes. Por lo tanto, el presente estudio analiza el uso de la secuencia de nucleótidos de la región control del ADN mitocondrial para evaluar la estructura genética intraespecífica de las poblaciones de truchas Yaqui en la zona de Mesa Tres Ríos, Sonora.

\section{MATERIALES Y MÉTODOS \\ Caracterización del área de estudio}

Las recolectas de ejemplares de truchas se realizó en arroyos de aguas frías en el área de Mesa Tres Ríos, Cuenca del Río Yaqui, en el extremo Noreste de Sonora en el bosque de coníferas de la Sierra Madre Occidental (Fig. 1). Los arroyos muestreados fueron el Arroyo La Presita, Arroyo Los Pescados, Arroyo La Cebadilla tributarios del Arroyo La Cueva y el Arroyo San Antonio tributario del Río Tres Ríos, además del Arroyo El Largo tributario de Arroyo El Arco, todos pertenecientes a la Subcuenca del Río Bavispe. La unión de estos afluentes en un solo cauce constituye el cauce del Río Bavispe. Adicionalmente, se recolectaron truchas nativas en el Arroyo El Mocho Bill de la Subcuenca de los Ríos Papigochic-Sirupa-Aros, contiguo al Arroyo El Largo, solo separados por el parteaguas del cerro La India. Los especímenes del Arroyo El Mocho Bill, a pesar de localizarse en una subcuenca distinta de la del Río Bavispe, se han incluido en este estudio debido a que son el producto de una la translocación de ejemplares de la trucha Yaqui provenientes del Arroyo El Largo (Francisco Fímbres, 2008. Comunicación Personal. Ejido El Largo, Sonora; Granja Trutícola, Ejido El Largo). Por esta razón, se reconocen dos áreas de muestreo, una a las localidades independientes mencionadas producto de la translocación (Área 1) y la otra a las localidades de truchas recolectadas en los otros arroyos que tienen conexiones geográficamente cercanas (Área 2).

\section{Recolecta de peces y sus muestras}

Las truchas fueron recolectadas durante cuatro salidas de campo en el mes de abril de 2001 y en los meses de mayo, julio y noviembre de 2008 amparadas por los permisos de recolecta DGOPA.02191.110309.0667 y SGPA/DGVS/00505/10 otorgados al autor para correspondencia por la SAGARPA y SEMARNAT, respectivamente. Los peces fueron recolectados en los arroyos La Presita, La Cueva, La Cebadilla, San Antonio, 


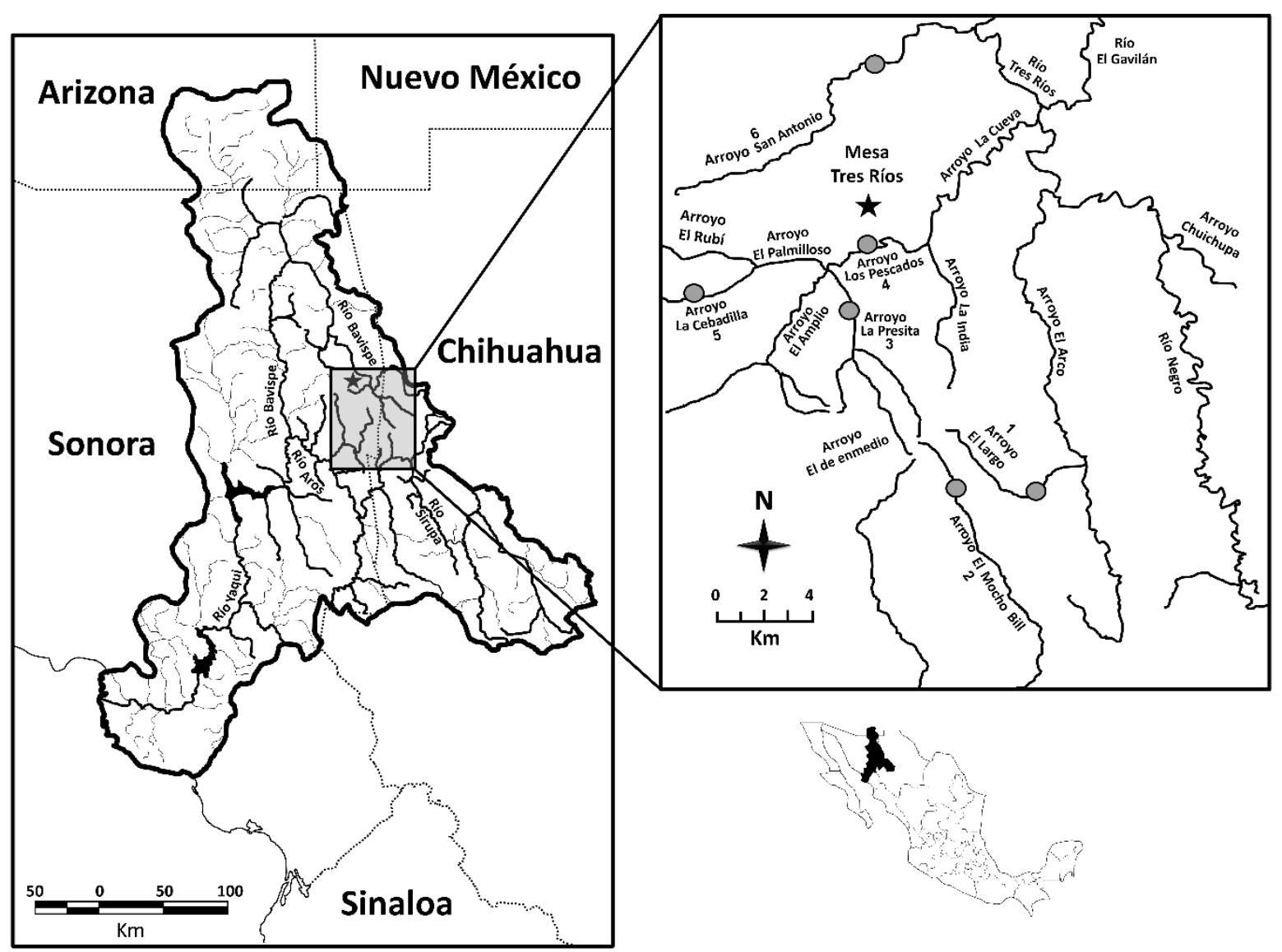

Figura 1. Localidades de recolecta para los ejemplares de trucha Yaqui en la Región de Mesa Tres Ríos, Sonora. Los círculos grises representan las localidades de recolecta en los arroyos muestreados, los números corresponden con las localidades de la tabla 1 y la estrella indica el poblado de Mesa Tres Ríos.

Figure 1. Collection locations for Yaqui trout in the Mesa Tres Rios Region, Sonora. Gray circles represent collection sites in the sampled streams, numbers correspond to localities in Table 1, and star indicates the town of Mesa Tres Ríos, Sonora.

El Largo y El Mocho Bill (Tabla 1) utilizando un equipo de pesca eléctrica (Coffelt BP-6) cubriendo los diferentes hábitats disponibles. Se tomaron muestras de tejido de la aleta pélvica de cada pez y se fijaron con etanol absoluto etiquetándolas individualmente para posteriormente ser depositadas a $-20^{\circ} \mathrm{C}$ en la Colección de Tejidos del Laboratorio de Ecología Molecular del Departamento de Investigaciones Científicas y Tecnológicas de la Universidad de Sonora (DICTUS) en Hermosillo. De igual forma cada pez previamente muestreado para tejido fue etiquetado individualmente y fijado en formol al $10 \%$ y depositados en la Colección de Peces Nativos del DICTUS y en el Laboratorio de Vertebrados de la Universidad Autónoma de Baja California (UABC) en Ensenada.

\section{PCR y análisis de secuencias}

El ADN genómico se extrajo de los tejidos de aletas siguiendo las instrucciones del Kit comercial QIAamp DNA Mini Kit de QIAGEN. Se amplificó la totalidad de la región control del ADN mitocondrial utilizando una PCR de punto final con los oligonucleótidos FW-LN20:5'-ACCACTAGCACCCAAAGCTA-3' y RV-HN20:5'-GTGTTATGCTTTAGTTAAGC-3', diseñados a partir de secuencias de la región control de otras truchas depositadas en el GenBank. Se utilizó un programa de amplificación de la PCR con los siguientes pasos, una desnaturalización inicial por 5 minutos a $94^{\circ} \mathrm{C}$ seguida de 34 ciclos de 50 segundos a $94^{\circ} \mathrm{C}, 1$ minuto a $50^{\circ} \mathrm{C}$ y 1 minuto a $72^{\circ} \mathrm{C}$ con una extensión final a $72^{\circ} \mathrm{C}$ por 10 minutos. Los productos de PCR fueron visualizados en geles de agarosa (Sigma-Aldrich) al $1 \%$ y fueron enviados para su secuenciación en ambos sentidos a Macrogen, Inc. (Seul, Corea).

Las secuencias obtenidas fueron editadas utilizando el programa Chromas Pro 1.41 (Technelysium Pty Ltd, South Brisbane, Queensland, Australia) y alineadas por medio del programa ClustalW (Thompson et al., 1994). El programa BLAST (Altschul et al., 1990) se utilizó para comparar las secuencias obtenidas con las secuencias de otras truchas depositadas en el GenBank. Para confirmar la monofilia de las secuencias obtenidas contra las de otras truchas del género Oncorhynchus se realizó un análisis filogenético de Máxima Verosimilitud (ML) a partir del modelo evolutivo TVR+G obtenido mediante las pruebas de tasa jerárquica de verosimilitud (hLRTs, por sus siglas en inglés) y el criterio de información de Akaike (AIC, por sus siglas en inglés; Posada y Buckley, 2004). El modelo se utilizó para calcular los valores 
y la topología del árbol a partir de una búsqueda de ML utilizando el intercambio de ramas (branch-swapping search) para un árbol de alta verosimilitud con los parámetros recuperados en el programa PAUP* (Swoford, 2002). El árbol fue utilizado para un análisis de bootstrap no paramétrico de 1000 pseudoréplicas para estimar el soporte de nodos en el análisis de ML. El árbol fue editado en el programa FigTree versión 1.3.1.

La heterogeneidad de las frecuencias haplotípicas fue medida con una prueba de $X^{2}$ con aleatorización de Monte Carlo utilizando el programa CHI2MCS (Danzmann y Ihssen, 1995). Se utilizó el programa Arlequin 3.0 (Excoffier et al., 2005) para realizar el análisis de diversidad de genes, número de loci analizados, sitios polimórficos (S), diversidad nucleotídica, frecuencia haplotípica y análisis molecular de varianza (AMOVA). El valor del índice de fijación (Fst) obtenido en la AMOVA se probó mediante la hipótesis Fst $=0$ usando la fórmula $\mathrm{x}^{2}=2$ NFst $(k-1)$, con g.1. $=(k-1)(s-1)$, en donde $k$ es el número de alelos diferentes, $N$ el número de organismos y s el número de muestras (Workman y Niswander, 1970). Se estimó el número de migrantes por generación de acuerdo a $N_{e} m=\left(F s t^{-1}-1\right) / 2($ Hartl, 1988). Debido a que las truchas recolectadas en el Arroyo El Mocho Bill fueron translocadas de la localidad de Arroyo El Largo, estas dos localidades se reconocen como una sola área de muestreo y se compara con las recolectadas en los otros arroyos.

\section{RESULTADOS Y DISCUSIÓN}

Se obtuvieron 61 secuencias de la región control completa del ADN mitocondrial con una longitud de 1000 a 1004 pb (Tabla 1). La similitud de estas secuencias con las depositadas en el GenBank para la región control de otras truchas del género Oncorhynchus, mostraron una homología de 99 \% y el análisis filogenético de ML realizado descubrió

Tabla 1.- Localidades de recolecta, número de acceso al GenBank y datos generales de los especímenes de trucha Yaqui recolectados en Sonora. USON = Universidad de Sonora, Elevación = metros sobre el nivel del mar.

Table 1.- Collection localities, GenBank accession numbers, and data of Yaqui trout specimens collected in Sonora. USON = University of Sonora, Elevation $=$ meters above sea level.

\begin{tabular}{|c|c|c|c|c|c|}
\hline Localidad & $\begin{array}{c}\text { Fecha de recol- } \\
\text { ecta }\end{array}$ & $\begin{array}{c}\text { Numero de catálogo y } \\
\text { espécimen }\end{array}$ & $\begin{array}{l}\text { Coordenadas } \\
\text { geográficas y } \\
\text { elevación }\end{array}$ & GenBank & $\begin{array}{c}\text { Número de secuencias } \\
\text { analizadas }\end{array}$ \\
\hline \multicolumn{6}{|l|}{ Área 1} \\
\hline $\begin{array}{l}\text { Arroyo El Largo, } 5 \mathrm{~km} \text { al } \\
\text { Este del Ejido Arroyo Lar- } \\
\text { go, } n=26\end{array}$ & $16-05-08$ & $\begin{array}{l}\text { USON-1108-11 } \\
\text { USON-1108-13 } \\
\text { USON-1227-1 } \\
\text { USON-1227-3 } \\
\text { USON-1227-5 } \\
\text { USON-1227-9 } \\
\text { USON-1227-15 }\end{array}$ & $\begin{array}{c}29^{\circ} 43^{\prime} 42.2^{\prime \prime} \mathrm{N} \\
108^{\circ} 37^{\prime} 29.4^{\prime \prime} \mathrm{O} \\
2200 \mathrm{msnm}\end{array}$ & $\begin{array}{l}\text { MK497757 } \\
\text { MK497756 } \\
\text { MK497745 } \\
\text { MK497744 } \\
\text { MK497740 } \\
\text { MK497750 } \\
\text { MK497752 }\end{array}$ & 26 \\
\hline \multirow[t]{2}{*}{$\begin{array}{l}\text { Arroyo El Mocho Bill al } \\
\text { cruce con el camino que } \\
\text { baja por la vertiente su- } \\
\text { reste del cerro la India } \\
\text { (Subcuenca del Río Aros), } \\
\mathrm{n}=10\end{array}$} & 03-11-08 & $\begin{array}{l}\text { USON-1230-7 } \\
\text { USON-1230-8 } \\
\text { USON-1230-10 } \\
\text { USON-1230-11 } \\
\text { USON-1230-12 }\end{array}$ & $\begin{array}{l}29^{\circ} 43^{\prime} 36.3^{\prime \prime} \mathrm{N} \\
108^{\circ} 40^{\prime} 22^{\prime \prime} \mathrm{O} \\
2000 \mathrm{msnm}\end{array}$ & $\begin{array}{l}\text { MK497753 } \\
\text { MK497751 } \\
\text { MK497741 } \\
\text { MK497746 } \\
\text { MK497743 }\end{array}$ & 10 \\
\hline & & Total & & & 36 \\
\hline \multicolumn{6}{|l|}{ Área 2} \\
\hline $\begin{array}{l}\text { Arroyo La Presita, tributa- } \\
\text { rio del Arroyo La Cueva, } \\
\text { justo en su unión con el } \\
\text { arroyo Amplio, } 200 \text { m co- } \\
\text { rriente arriba, } n=12\end{array}$ & $\begin{array}{l}15-05-08 \\
23-04-01\end{array}$ & $\begin{array}{l}\text { USON-1105-1 } \\
\text { USON-1105-4 } \\
\text { USON-1140-1 }\end{array}$ & $\begin{array}{c}29^{\circ} 48^{\prime} 32.8^{\prime \prime} \mathrm{N} \\
108^{\circ} 43^{\prime} 12.6^{\prime \prime S} \\
1640 \mathrm{msnm}\end{array}$ & $\begin{array}{l}\text { MK497754 } \\
\text { MK497747 } \\
\text { MK497742 }\end{array}$ & 12 \\
\hline $\begin{array}{l}\text { Arroyo Los Pescados, ca. } \\
\text { de Rancho La Primavera, } \\
\text { tributario del Arroyo La } \\
\text { Cueva, } n=9\end{array}$ & 25-04-01 & $\begin{array}{l}\text { UABC-1379-1 } \\
\text { UABC-1379-2 }\end{array}$ & $\begin{array}{c}29^{\circ} 48^{\prime} 57.2^{\prime \prime} \mathrm{N} \\
108^{\circ} 40^{\prime} 48.1^{\prime \prime} \mathrm{S} \\
1571 \mathrm{msnm}\end{array}$ & $\begin{array}{l}\text { MK497749 } \\
\text { MK497755 }\end{array}$ & 9 \\
\hline $\begin{array}{l}\text { Arroyo La Cebadilla, arro- } \\
\text { yo arriba desde su unión } \\
\text { con el Arroyo El Palmillo- } \\
\text { so, } n=2\end{array}$ & 09-07-08 & USON-1154-1 & $\begin{array}{l}29^{\circ} 49^{\prime} 11.3^{\prime \prime} \mathrm{N} \\
108^{\circ} 44^{\prime} 4.9^{\prime \prime} \mathrm{O} \\
2200 \mathrm{msnm}\end{array}$ & MK497748 & 2 \\
\hline \multirow[t]{2}{*}{$\begin{array}{l}\text { Arroyo San Antonio arri- } \\
\text { ba de Rancho San Anto- } \\
\text { nio, } n=2\end{array}$} & 23-04-01 & UABC-1378-1 & $\begin{array}{c}29^{\circ} 52^{\prime} 46.5^{\prime \prime} \mathrm{N} \\
108^{\circ} 44^{\prime} 03.6^{\prime \prime} \mathrm{S} \\
1610 \mathrm{msnm}\end{array}$ & MK497758 & 2 \\
\hline & & Total & & & 25 \\
\hline
\end{tabular}


la monofilia entre las truchas de la región de Mesa Tres Ríos y su afinidad como la trucha arcoíris y con otras especies del género Oncorhynchus (Fig. 2). Varios autores indican que la trucha Yaqui está más emparentada filogenéticamente con la trucha arcoíris que con otras truchas nativas de América (Loudenslager et al., 1986; Nielsen, 1996, 1997; Nielsen et al., 1997, 1998) y que pertenece a un complejo de truchas nativas donde se reconocen varios linajes genéticos, que podrían considerarse a nivel de especie (Nielsen y Sage, 2001; Camarena-Rosales et al., 2008; De Los Santos-Camarillo, 2008; Mayden et al., 2010; Abadía-Cardoso et al., 2015), donde estudios a mayor profundidad sitúan a la trucha Yaqui que habita la subcuenca del Río Bavispe en el linaje más norteño junto con las poblaciones de las cuencas de los ríos Casas Grandes y Mayo y se considera una ESU que debe describirse como nueva especie para la ciencia y desarrollar las prácticas de conservación necesarias para asegurar su sobrevivencia (García de León, en preparación).

El análisis de la distribución de las frecuencias haplotípicas agrupadas por áreas geográficas mostró un total de nueve haplotipos, tres fueron compartidos en ambas áreas geográficas y de los seis restantes, cuatro se encontraron en el área 1 (Arroyos El Largo y El Mocho Bill) y dos en el área 2 (Arroyos La Presita, Los Pescados y La Cebadilla) de manera independiente (Tabla 2). Cuatro haplotipos fueron únicos para su localidad, encontrándose dos en el área 1 (haplotipos 7 y 8 ) y dos en el área 2 (haplotipos 6 y 9). El análisis de secuencias muestra a los valores más altos de diversidad genética en las poblaciones de los arroyos El Largo y El Mocho Bill (Área 1) con una media y desviación estándar de $0.7159 \pm 0.0678, p=$ 0.05), mientras que en los arroyos La Presita, Los Pescados y
La Cebadilla (Área 2) mostraron un valor de $0.3768 \pm 0.1224$, $p=0.05)$. El valor promedio para todas las poblaciones fue $0.5463 \pm 00.0951$. Los valores de la diversidad nucleotídica para el área 2 fueron los mayores con $0.0047 \pm 0.0026, p=$ 0.05 ) y para el área 1 de $0.0016 \pm 0.0011, p=0.05$ ), mientras que para el total de las poblaciones fue de $0.0031 \pm 0.0018$. El análisis de la diversidad molecular muestra 1004 loci para el total de las secuencias analizadas, debido a que la posición de cada uno de los nucleótidos corresponde a un locus. Así, tenemos que nueve loci polimórficos se obtuvieron del área geográfica 1 y 32 del área 2, con un valor promedio de 20.5 y un número de haplotipos de 7 y 5 , respectivamente (Tabla 3 ). Camarena Rosales et al. (2008) menciona que las truchas nativas de la zona del Río Yaqui y otros miembros del complejo en la SMO, así como la trucha de San Pedro Mártir en Baja California (O. mykiss nelsoni) presentan haplotipos exclusivos que pueden estar relacionados con el stock de origen del cual provienen. La existencia de haplotipos compartidos entre individuos de los arroyos El Largo y el Mocho Bill corresponde a la translocación reportada por cultivadores de truchas locales y la ausencia de los haplotipos 7 y 8 en los especímenes del Arroyo El Mocho Bill se debe al limitado número de individuos (de 6 a 8) utilizados en la translocación. La presencia de haplotipos comunes y exclusivos en individuos de estas poblaciones que habitan en arroyos aislados de la zona de Mesa Tres Ríos en Sonora, así como los valores de diversidad genética, nucleotídica y los polimorfismos detectados, puede deberse a las diferentes historias de aislamiento geográfico, como lo reportan Camarena-Rosales et al. (2008) para truchas de la SMO.

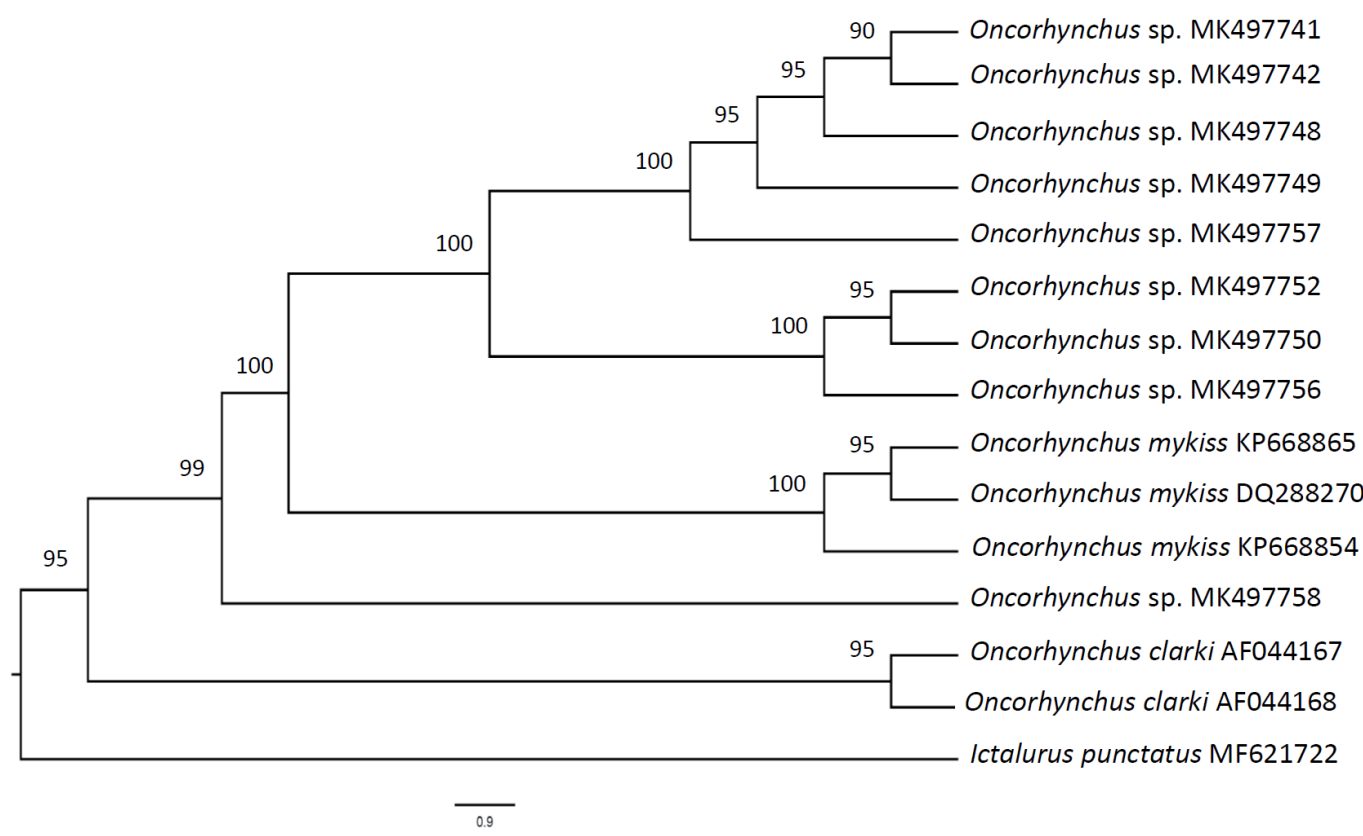

Figura 2. Cladograma de Máxima Verosimilitud para la nueve haplotipos de trucha Yaqui observados en la región de Mesa Tres Ríos, Sonora. Los números por arriba de los nodos indican el porcentaje de remuestreo no paramétrico.

Figure 2. Maximum Likelihood Cladogram for the nine Yaqui trout haplotypes observed in the Mesa Tres Rios region, Sonora. Numbers above nodes indicate the percentage of nonparametric boostrap. 
Tabla 2. Distribución de las frecuencias de haplotipos en las localidades de muestreo en Mesa Tres Ríos, Sonora. Table 2. Haplotype frequencies distribution in sampling locations in Mesa Tres Rios, Sonora.

\begin{tabular}{|c|c|c|c|c|c|c|}
\hline \multicolumn{3}{|c|}{ Área 1} & \multicolumn{4}{|c|}{ Área 2} \\
\hline Haplotipos & El Largo & El Mocho Bill & La Presita & La Cebadilla & San Antonio & Los Pescados \\
\hline 1 & 3 & 1 & & & & \\
\hline 2 & 1 & 1 & 2 & & & \\
\hline 3 & 12 & 6 & 9 & 2 & & 8 \\
\hline 4 & 5 & 1 & & & & \\
\hline 5 & 1 & 1 & 1 & & & \\
\hline 6 & & & & & & 1 \\
\hline 7 & 3 & & & & & \\
\hline 8 & 1 & & & & & \\
\hline 9 & & & & & 2 & \\
\hline Total & 7 & 5 & 3 & 1 & 1 & 2 \\
\hline
\end{tabular}

Tabla 3. Índices de diversidad molecular para los haplotipos de las localidades de muestreo en las dos áreas geográficas en la región de Mesa Tres Ríos, Sonora.

Table 3. Haplotypes molecular diversity indexes of sampling locations in the two geographical areas in the Mesa Tres Rios region, Sonora.

\begin{tabular}{lccccc}
\hline & $\begin{array}{c}\text { Número de copias } \\
\text { de gen }\end{array}$ & $\begin{array}{c}\text { Número de } \\
\text { haplotipos }\end{array}$ & Número de loci & $\begin{array}{c}\text { Número de loci } \\
\text { útiles }\end{array}$ & $\begin{array}{c}\text { Número de sitios } \\
\text { polimórficos }\end{array}$ \\
\hline Área 1 & 36 & 7 & 1003 & 1003 & 9 \\
Área 2 & 25 & 5 & 1004 & 1004 & 32 \\
\hline
\end{tabular}

La distribución de las frecuencias de haplotipos mostró 44 de los 61 especímenes analizados en tres haplotipos compartidos $(2,3$ y 5$)$ en al menos cuatro de las seis localidades muestreadas de ambas áreas geográficas. Este número de haplotipos distribuidos a lo largo de los sitios de muestro en ambas áreas estudiadas puede deberse a la migración natural de la trucha en eventos reproductivos en la zona utilizando su conexión con el Río Negro y el Río Tres Ríos (Fig. 1), que representan los cursos de agua donde los arroyos estudiados desembocan. Otros autores han reportado el movimiento de algunas truchas, con fines de desove durante la primavera, en distancias largas hasta de $50 \mathrm{~km}$ (Thurow et al., 1988; Henderson et al., 2000). Sin embargo, los eventos de translocación de truchas nativas por los truticultores locales en el pasado y del cual aún no se tiene la lista completa de estas acciones, puede ser el mecanismo de flujo de genes entre las poblaciones de esta remota área.

El análisis de AMOVA para determinar la estructura genética indica que el $91.96 \%$ del total de la variación ocurre dentro de las poblaciones, mientras que el $8.04 \%$ ocurre entre las poblaciones. El valor de Fst (0.08035) de acuerdo con la prueba de $\mathrm{x}^{2}$ indica un muy bajo nivel de diferenciación genética no distinta de cero entre las dos áreas geográficas analizadas (Tabla 4). El número de migrantes por generación (Nem) considerando el valor de Fst obtenido fue de alrededor de 5.72 individuos por generación. La comparación de estos resultados y el análisis de diversidad genética con otros estudios reportados no muestran evidencia de pérdida genética dentro de la población en el Sitio 2 del área estudiada (Camarena-Rosales et al., 2008). Nuestros resultados no permiten inferir una fragmentación aparente del hábitat, adicionalmente no existe evidencia de sobrepesca de truchas en esa región. Sin embargo, otros estudios en truchas del género Oncorhynchus han demostrado que el flujo de genes tiene más influencia que la deriva genética en poblaciones con distancias geográficas cortas y esto resulta en una población panmíctica. La falta de diferenciación genética en la truchas Yaqui de las poblaciones del área de Mesa Tres

Tabla 4. Indicadores del análisis molecular de varianza (AMOVA) para los dos sitios geográficos de muestreo en Mesa Tres Ríos, Sonora.

Table 4. Indicators of molecular analysis of variance (AMOVA) for the two geographic sampling sites in Mesa Tres Rios, Sonora.

\begin{tabular}{lcccc}
\hline Fuente de la variación & g. I. & Suma de cuadrados & Varianza & $\begin{array}{c}\text { Porcentaje de } \\
\text { variación }\end{array}$ \\
\hline Entre las poblaciones & 1 & 1.022 & $0.02540 \mathrm{Va}$ & 8.04 \\
Dentro de las poblaciones & 58 & 16.861 & $0.29071 \mathrm{Vb}$ & 91.96 \\
Total & 59 & 17.883 & 0.31611 & \\
Índice de Fijación Fst $=0.08035$ & & & & \\
\hline
\end{tabular}


Ríos en Sonora también puede deberse a un ancestro común reciente (Cegelski et al., 2006). Sin embargo, hay otros factores que pueden causar la falta de variación genética en esta región, como la ausencia de presiones ambientales selectivas y la presencia de dispersión natural dentro de la población (Gresswell et al., 1997).

Algunos estudios han documentado la diferenciación genética entre poblaciones dentro de la misma cuenca por la separación de barreras geográficas (Carlsson et al., 1999). Sin embargo, en el área de estudio de este trabajo, no se ha detectado hasta la fecha ninguna barrera natural o estructura física antropogénica que sirva para este propósito. A pesar de que las temperaturas en las épocas cálidas del año pueden funcionar como barreras para esta especie de aguas frías, no existen datos suficientes de la temperatura para ser comparados y determinar si funcionan como barrera térmica. Aparentemente, el bajo grado de diferenciación genética ( Fst $=0.08035)$ y el número de migrantes por generación obtenido en este estudio ( $\mathrm{Nem}=5.72$ ) puede deberse a un alto flujo de genes (Wenburg et al., 1998) en ausencia de barreras geográficas que impiden el flujo entre la trucha, además de la dispersión y la falta de presiones ambientales selectivas (Gresswell et al., 1997). Estos resultados pueden indicar que el proceso de migración es masivo en un corto período de tiempo, lo que sugiere que el papel de la translocación entre la trucha Yaqui en diferentes corrientes puede ser una práctica más común de lo que se podría pensar en el pasado reciente. No se detectó estructura genética de las poblaciones de la trucha en las localidades muestreadas en Sonora en el área de Mesa Tres Ríos, por lo tanto, se considera como una sola población que ocupa corrientes de agua fría en la región.

Por último, a pesar de los avances en las técnicas de estudio de la variabilidad genética en la actualidad, el análisis de secuencia nucleotídica de la región de control del ADN mitocondrial de la trucha Yaqui representa una herramienta muy útil y valiosa para describir su variabilidad genética y estructura poblacional. La población de truchas Yaqui de Sonora en la zona de Mesa Tres Ríos representa una ESU que requiere protección, conservación y además resulta un recurso útil para el desarrollo acuícola local.

\section{CONCLUSIONES}

El análisis de variabilidad genética realizado a 61 secuencias de la región control del ADN mitocondrial de truchas Yaquis provenientes de Mesa Tres Ríos, Sonora no detectó estructura genética poblacional, por lo tanto se considera la existencia de una sola población en esa región. Tres haplotipos fueron detectados en ambas vertientes, el resto de los seis haplotipos representan individuos distribuidos en los seis arroyos de muestreo. No se detectó evidencia de extracción por pesca sobre la población de trucha Yaqui en la región de Mesa Tres Ríos.

Se documenta la translocación de individuos de la trucha Yaqui desde el Arroyo El Largo en la subcuenca del Río Bavispe al Arroyo El Mocho Bill de la subcuenca de los Ríos
Papigochic - Sirupa - Aros, ambos de la cuenca del Río Yaqui. El análisis de la secuencia de la región control del ADN mitocondrial de la trucha Yaqui representa una herramienta muy útil y valiosa para la descripción de su variabilidad genética y la estructura poblacional. La población de trucha Yaqui en la región de Mesa Tres Ríos en Sonora representa una unidad evolutiva susceptible de manejo para su protección, conservación y uso como recurso acuícola.

\section{AGRADECIMIENTOS}

Agradecemos al Instituto de Acuacultura del Estado de Sonora por el apoyo económico para el desarrollo de este trabajo. Al Sr. Ramón Fímbres y su familia en el Ejido El Largo por brindar apoyo, alojamiento e información durante las recolectas. A la Licenciatura en Biología de la UNISON por su apoyo. A María Irais Trujillo-Villalba, Leonides Loera-Urías, Francisco Humberto Monteverde-Valenzuela, Leonardo Varela-Espinoza, Jairo Contreras-Encinas por su apoyo en las recolectas.

\section{REFERENCIAS}

AbadíaCardoso, A., Garza, J.C., Mayden, R.L. y García de León, F.J. 2015. Genetic Structure of Pacific Trout at the Extreme Southern End of Their Native Range. PLoS ONE. 10(10): p.e0141775.

Abadía-Cardoso, A., García-De León, F.J. y Garza, J.C. 2016. Historia evolutiva y biodiversidad genética de las truchas de la Sierra Madre Occidental. En: La trucha dorada mexicana. A. Ruiz-Luna y F.J. García-De León (eds.), pp 29-38. CIAD,CIB, CONACYT, La Paz, BCS.

Altschul, F., Gish, G., Miller, W., Myers, E.W. y Lipman, D.J. 1990. Basic local alignment search tool. Journal of Molecular Biology. 215:403-410.

Behnke, R.J. 1991. Mexican golden trout Oncorhynchus chrysogaster. En:Trout. J. Stolz y J. Schnell (eds.), pp. 300-303. The Wild Life Series. Stackpole Books, Harrisburg, PA.

Behnke, R.J. 2002. Trout and Salmon of North America. The Free Press, New York.

Camarena-Rosales, F., Ruiz-Campos, G., De La Rosa-Vélez, J., Manden, R., Hendrickson, D.A., Varela-Romero, A. y García De León, F. 2008. Mitochondrial haplotype variation in wild trout populations (Teleostei: Salmonidae) from northwest Mexico. Reviews in Fish Biology and Fisheries. 18:33-45.

Carlsson, J., Olsen, H.K., Nilsson, J., Overli, O. y Stabell, O. B. 1999. Microsatellites reveal fine-scale genetic structure in streamliving brown trout. Journal of Fish Biology. 55:1290-1303.

Cegelski, C.C., Campbell, M. R., Meyer, K.A. y Powell, M.S. 2006. Multiscale genetic structure of Yellowstone Cutthroat trout in the upper Snake River basin. Transactions of the American Fisheries Society. 135:711-726.

Danzmann, R.G. y lhssen, P. E. 1995. A phylogeographic survey of brook charr (Salvelinus fontinalis) in Algonquin Park, Ontario based upon mitochondrial DNA variation. Molecular Ecology. 4:681-697.

De los Santos-Camarillo, A.B. 2008. Definición de unidades taxonómicas en el complejo de truchas del noroeste de México, mediante el análisis de marcadores microsatálites. Tesis de Maestría. Centro de Investigaciones Biológicas del Noroeste, S.C., La Paz, Baja California Sur. 
Espinosa-Pérez, H., García de León, F. G. Ruiz, Varela-Romero, A., Barriga-Sosa, I., Arredondo-Figueroa J., Hendrickson, D.A., Camarena-Rosales, F. y De los Santos-Camarillo, A. 2007. Las Truchas Mexicanas. Especies. 16:9-14.

Escalante, M.A., Javier García-De León, F., Dillman, C.B., De los Santos Camarillo, A.B., George, A. y Barriga Sosa, I.A. 2016. Introgresión genética de la trucha arcoíris exótica en poblaciones de trucha dorada mexicana. En: La Trucha Dorada Mexicana. A. Ruiz-Luna y F. J. García-De León (eds.), pp 125-136. CIAD, CIB, CONACYT, La Paz, BCS.

Escalante, M.A., García-De-León, F.J., Dillman, C.B. de los SantosCamarillo, A., George, A., de los A. Barriga-Sosa, I. Ruiz-Luna, A, Mayden, R.L. y Manel, S. 2014. Genetic introgression of cultured rainbow trout in the Mexican native trout complex. Conservation Genetics. 15:1063-1071.

Evermann, B.W. 1908. Descriptions of a new species of trout (Salmo nelsoni) and a new cyprinodont (Fundulus meeki) with notes on other fishes from Lower California. Proceedings of the Biological Society of Washington. 21:19-30.

Excoffier, L., Laval, G. y Schneider, S. 2005. Arlequin ver. 3.0: An Integrated software package for population genetics data analysis. Evolutionary Bioinformatics. 1:47-50.

FAO. 2005. Cultured Aquatic Species Information Programme. Oncorhynchus mykiss. Text by Cowx, I. G. In: FAO Fisheries and Aquaculture Department [online]. Rome. Updated 15 June 2005. [Consultado 25 Enero 2019]. Disponible en: http:// www.fao.org/fishery/culturedspecies/Oncorhynchus_ mykiss/en

García-De León, F.J., Dillman, C.B., De los Santos-Camarillo, A.B., George, A.L., Camarena-Rosales, F., Barriga-Sosa, I. de los A. y Mayden, R.L. En preparación. First steps towards the identification of Evolutionarily Significant Units in Mexican native trout: an assessment of microsatellite variation. En preparación.

Gresswell, R.E., Liss, W.J., Larson, G.L. y Bartlein, P.J. 1997. Influence of basin- scale physical variables on life history characteristics in Yellowstone Lake. North American Journal of Fisheries Management. 17:1046-1064.

Hartl, D.L. 1988. A Primer of Population Genetics. Sinauer Associates, Inc. Sunderland, Massachusetts.

Henderson, R., Kershner, J.L. y Toline, C.A. 2000. Timing and location of spawning by nonnative wild Rainbow trout and native Cutthroat trout in the south fork Snake River, Idaho, with implications for hybridization. North American Journal of Fisheries Management. 20:584-596.

Hendrickson, D.A., Espinosa-Pérez, H., Findley, L.T., Forbes, W., Tomelleri, J.R., Mayden, R.L., Nielsen, J.L., Jensen, B., Ruiz-Campos, G., Varela-Romero, A., Van der Heiden, A., Camarena-Rosales, F. y García de León, F. 2002. Mexican native trouts: a review of their history and current systematic and conservation status. Review in Fish Biology and Fisheries. 12:273-316.

Hendrickson, D.A., Neely, D.A., Mayden, R.L., Anderson, K., Brooks, J.E., Camarena-Rosales, F., Cutter, R., Cutter, L., De Los Santos C., A.B., Ernsting, G.W., Espinoza-Pérez, H., Findley, L.T., García-De León, F.J., George, A.L., Hatch, J., Kuhajda, B.R., Mayden, K.E., Mcnyset, K., Nielsen, J.L., Pfeifer, F.W., Propst, D.L., Ruiz-Campos, G., St. Clair, E., Tomelleri, J.R. y VarelaRomero, A. 2006. Conservation of Mexican native trout and the discovery, status, protection and rediscovery of the Conchos trout, the first native. En: Studies of North American
Desert Fishes in Honor of E.P. (Phil) Pister, Conservationist. M.L. Lozano-Vilano y A.J. Contreras-Balderas (eds.), pp. 162201. Facultad de Ciencias Biológicas, Universidad Autónoma de Nuevo León. Monterrey, México.

Loudenslager, E.J., Rinne, J.N., Gall, G.A.E. y David, R.E. 1986. Biochemical genetic studies of native Arizona and NewMexico trout. Southwestern Naturalist. 31(2): 221-234.

Mayden, R.L. 2004. Biodiversity of Mexican trout (Teleostei: Salmonidae: Oncorhynchus): recent findings conservation concerns, and management recommendations. En: Homenaje al Doctor Andrés Reséndez-Medina. M.L. Lozano-Vilano y A.J. Contreras-Balderas, (eds.), pp 269-282. Universidad Autónoma de Nuevo León, Monterrey, México.

Mayden, R.L., Dillman, C.B., Espinosa-Pérez, H., Tomelleri, J.R., Kuhajda, B.R., Hendrickson, D.A., Ruiz-Campos, G., De los Santos-Camarillo, A.B., García De León, F., Varela-Romero, A., Propst, D.L., Brooks, J.E., Findley, L.T., George, A.L., Neely, D.A., Barriga-Sosa, I.A. y Mayden, K.E. 2010. Evolution and Diversity of Trout Species in México. En: R.F. Carline y C. Lo Sapio, (eds.), pp 134-144. Proceedings of the X Wild Tout Symposium, Bozeman, Montana. September 28-39, 2010. West Yellowstone, MT.

Miller, R.R. 1950. Notes on the Cutthroat and Rainbow trout with the description of a new species from the Gila River, New Mexico. Museum of Zoology of the Michigan University. 529:1-43.

Miller R.R., Minckley, W.L. y Norris, S.M. 2005. Freshwater Fishes of Mexico. The University of Chicago Press, Chicago.

Minckley, W.L. y Marsh, P.C. 2009. Inland fishes of the greater Southwest: chronicle of a vanishing biota. University of Arizona Press, Tucson, AZ.

Needham, P.R. y Gard. R. 1964. A new trout from Central Mexico: Salmo chrysogaster, the Mexican golden trout. Copeia. 1964:169-173.

Nelson, E.W. 1921. Lower California and its natural resources. Memories of the National Academy of Science. 16:1-194.

Nielsen, J.L. 1996. Using mitochondrial and nuclear DNA to separate hatchery and wild stocks of rainbow trout in California and Mexico. En: Aquaculture Biodiversity Symposium Proceedings International Congress on the Biology of Fishes, Julio 14-18. E.M Donaldson y D.D. MacKinlay, (eds.), pp 139-147, San Francisco, California.

Nielsen, J.L. 1997. Molecular genetics and evolutionary status of the trout of the Sierra Madre. En: Wild trout Vl: Putting the native back in wild trout. R.E. Gresswell, P. Dwyer y. R. H. Hamre, (eds.), pp 103-109. Montana State University, Bozeman, Montana.

Nielsen, J.L. y Sage, G.K. 2001. Microsatellite analyses of the trout of northwest Mexico. Genetica. 111:269-278.

Nielsen, J.L., Fountain, M.C. y Wright, J.M. 1997. Biogeographic analysis of Pacific trout (Oncorhynchus mykiss) in California and Mexico based on mitochondrial DNA and nuclear microsatellites. En: Molecular Systematics of Fishes. T.D. Kocher y C.A. Stepien, (eds.) pp 53-73. Academic Press. San Diego, California.

Nielsen, J.L., Fountaun, M.C., Campoy-Favela, J.R., Cobble, K. y Jensen, B.L. 1998. Oncorhynchus at the southern extent of their range: A study of mtDNA control-region sequence with special reference to an undescribed subspecies of $O$. mykiss from Mexico. Environmental Biology of Fishes. 51:7-23. 
Posada, D. y Buckley T.R. 2004. Model selection and model averaging in phylogenetics: advantages of Akaike information criterion and Bayesian approaches over likelihood ratio tests. Systematic Biology. 53:793-808.

Ruiz-Campos, G. y Pister, E.P. 1995. Distribution, habitat, and current status of the San Pedro Martir Rainbow trout, Oncorhynchus mykiss nelsoni. Bulletin of South California Academy of Science. 94:131-148.

Ruiz-Campos, G., Camarena-Rosales, F., Varela-Romero, A., Sánchez-González, S. y De la Rosa-Vélez, J. 2003. Morphometric variation of native trout populations from Northwestern Mexico (Pisces: Salmonidae) Review in Fish Biology and Fisheries. 13:91-110.

Smith, R.H. 1991. Rainbow trout, Oncorhynchus mykiss. En: Trout. The wildlife series trout. J. Stolz y J. Schenell, (eds.), pp 304-322. Stackpole Books, Harrisburg.

Snyder, J.O. 1926. The trout of the Sierra San Pedro Martir, Lower California. University of California Publications in Zoology. 21:419-426.

Swoford, D.L. 2002. PAUP* 4.0: Phylogenetic analysis using parsimony and other methods. Sunderland, MA, Sinauer Associates.
Thompson J.D., Higgins, D.G. y Gibson, T.J. 1994. CLUSTAL W: Improving the sensitivity of progressive multiple sequence alignment through sequence weighting, position-specific gap penalties and weight matrix choice. Nucleic Acid Research. 22:4673-4680.

Thurow, R.F., Corsi, C.E. y Moore, V.K. 1988. Status, ecology, and management of Yellowstone Cutthroat trout in the upper Snake River drainage, Idaho. En: Status and management of interior stocks of Cutthroat trout. R.E. Gresswell, (ed.), pp 25-36 American Fisheries Society, Symposium 4, Bethesda, Maryland.

Varela-Romero, A. y Hendrickson, D.A. 2009. Los peces dulceacuícolas de Sonora. En: Diversidad Biológica de Sonora. F. Molina-Fraener y T. Van Devender, (eds.). UNAM, México.

Wenburg, J.K., Bentzen, P. y Foote, C.J. 1998. Microsatellite analysis of genetic population structure in an endangered salmonid: the coastal Cutthroat trout (Oncorhynchus clarki clarki). Molecular Ecology. 7:733-749.

Workman, P.L. y Niswander, J.D. 1970. Population studies on Southwestern Indian tribes. II. Local genetic differentiation in the Papago. American Journal of Human Genetics. 22:2429. 